\title{
Introduction to Health Behavior Change Support Systems 2022
}

\author{
Khin Than Win \\ University of Wollongong \\ Australia \\ win@uow.edu.au
}

\author{
Amanda Blok \\ University of Michigan \\ United States of America \\ acblok@umich.edu
}

\author{
Harri Oinas-Kukkonen \\ University of Oulu \\ Finland \\ harri.oinas-kukkonen@oulu.fi
}

More than ever, behaviour change perspectives play a significant role in healthcare management and personalized care. Health behavior change support systems (HBCSS) assist in disease prevention, health promotion and healthcare management [1]. To date, many studies have presented HBCSS perspectives [2], application of persuasive technologies in biomedical informatics $[3,4]$ health education, consumer engagement, and the effectiveness of HBCSS in healthcare [5-7].

For our 2022 mini-track, four peer-reviewed papers were accepted and further the science around HBCSS. The design and effect of digital technology, such as mobile devices, enabling interventions utilizing avatars, coping assistants, virtual coaches and telehealth on patient experience, engagement, selfcare and coping were studied. Strategies often targeted users for ongoing engagement for persistent or pervasive problems. Assisting users to engage in chronic condition management, mitigate stress, address obesity and subsequent use of telehealth for acquiring healthcare. Design decisions around enhancing self-presence for relevance, best practices for architectural and avatar design.

To address problematic user engagement, design in digital health interventions to assimilate a self-concept during human-computer interaction can enhance personal relevance through self-presence. Using these principles, Durneva and colleagues [8] tested key design elements of an avatar for enhanced selfpresence in a digital health intervention. Utilising design science research approach, their theory-driven design findings can assist in designing interventions for self-care of those with chronic conditions.

A pervasive problem of stress and coping could be addressed using readily available mobile technology. Mobile devices can not only facilitate collecting sensor data for monitoring, but also delivery of strategies for coping at the point of need. Schmidt and colleagues [9] present their design of architectural components and algorithm for selecting strategies of coping to present. They also discuss opportunities and challenges discovered through their studied prototype.

Understanding user preferences in design is necessary for building effective health behavior change interventions. Weimann and colleagues [10] explored these decisions in designing virtual coach for those who are morbidly obese. The design science research approach is adopted in the artefact design. Evaluation results and the design knowledge on developing Avatar as the virtual coach were presented.

Determining the effect of telehealth on patient experience has implications for future use and integration of this technology. Duane and colleagues [11] explore the influence of patient perceptions of their telehealth experience with use behaviors, clinical information recall, and satisfaction. They provide best practices for clinicians and researchers for integrating telehealth into healthcare.

Using an innovative combination of behavioural theory and mobile technology design, these papers present the contribution of HBCSS for disease prevention, including chronic condition management, stress, obesity and telehealth visits.

\section{References}

[1] Win K.T., Roberts M.R.H, Oinas-Kukkonen, H, Persuasive system features in computer-mediated lifestyle modification interventions for physical activity, Informatics for Health and Social Care, 2019. 44(4): p. 376- 404,

DOI: $10.1080 / 17538157.2018 .1511565$

[2] Oinas-Kukkonen, H., A foundation for the study of behavior change support systems. Personal and Ubiquitous Computing, 2013. 17(6): p. 1223-1235.

[3] Matthews, J., et al., Persuasive Technology in Mobile Applications Promoting Physical Activity: a Systematic Review. Journal of Medical Systems, 2016. 40(3): p. 1-13

[4] Win K.T., et al., Persuasive Systems Design features in Promoting Medication Management for consumers, Proceedings of the 50th Hawaii International Conference on System Sciences, 2017, (pp. 3326-3335). HICSS.

[5] Lehto T. \& Oinas-Kukkonen H. (2011) Persuasive Features in Web-Based Alcohol and Smoking Interventions: A Systematic Review of the Literature. Journal of Medical Internet Research, 13(3), e46. 
[6] Karppinen P., et al. (2016) Persuasive User Experience in Health Behavior Change Support System: A 12-month Study for Prevention of Metabolic Syndrome. International Journal of Medical Informatics, Vol. 96, December, pp. 51-61 [7] Meedya et al. Developing and testing a mobile application for breastfeeding support: The Milky Way application, Women and Birth, 2020, ISSN 1871-5192, https://doi.org/10.1016/j.wombi.2020.02.006.
[8] Durneva and LeRouge, Designing Personally Relevant Avatars for Digital Health Interventions: The Biocultural Perspective of Presence, in this issue.

[9] Schimdt et al. Towards Designing a Mobile Stress Coping Assistant, in this issue.

[10] Weimann T., et al., Peer Buddy or Expert? - On the Avatar Design of a Virtual Coach for Obesity Patients, in this issue.

[11] Duane et al., The Virtual Doctor is in: The Effect of Telehealth Visits on Patient Experience, in this issue. 